\title{
Importância da genética no serviço público: relato da extinção de um setor de genética no Município de São Paulo, Brasil
}

\author{
Lilian Maria José Albano ${ }^{1}$
}

RESUMO A disponibilidade de um serviço de genética em hospitais deveria ser uma questão de interesse público. A genética pode abreviar o período gasto na formulação de diagnósticos, reduzir o tempo médio de internação, antecipar a escolha de tratamentos adequados, impedir ou minimizar possíveis seqüelas e, desta forma, reduzir os custos. O objetivo do presente estudo foi descrever o fechamento, em 1996, do setor de genética do Hospital Infantil Menino Jesus, de São Paulo, SP, Brasil. Realizou-se uma análise retrospectiva do trabalho desenvolvido naquele setor entre 1992 e 1996, com ênfase na detecção de anormalidades cromossômicas. De todos os casos avaliados no período, 571 foram codificados em banco de dados. Alguma anormalidade cromossômica foi observada em $20 \%$ dos 350 cariótipos realizados. A presença de um serviço de genética em hospitais minimiza o surgimento de manifestações clínicas em portadores de distúrbios genéticos, melhora a qualidade de vida desses pacientes e permite que eles recebam informação sobre o risco de recorrência, além de evitar desperdícios decorrentes da realização inadequada de exames caros. Tais benefícios justificam o investimento necessário para que este tipo de serviço funcione.

O grande avanço da genética, nas últimas décadas, tem permitido a melhor compreensão de certas doenças e o conhecimento de muitas outras. Vários esforços, atendendo aos anseios de um número cada vez maior de especialidades, têm sido realizados para se detectar, o quanto antes, as malformações congênitas, os desvios metabólicos, as

\footnotetext{
${ }^{1}$ Universidade de São Paulo, Faculdade de Medicina. Endereço e pedidos de separatas devem ser enviados para o seguinte endereço: Unidade de Genética do Instituto da Criança, Hospital das Clínicas da Faculdade de Medicina da Universidade de São Paulo (ICr - HC-FMUSP), Avenida Dr. Enéas de Carvalho Aguiar 647, Cerqueira César, CEP 05403-000, São Paulo, SP. Telefone: +55-11-3069-8671; fax: +55-113069-8503; e-mail: lima@icr.honet.usp.br
}

doenças heredodegenerativas, as displasias esqueléticas e todos os distúrbios genéticos passíveis de um diagnóstico pré ou pós-natal.

Atualmente, verifica-se um grande e profícuo intercâmbio entre as várias especialidades médicas e outras áreas da saúde, resultando em um saldo final extremamente positivo. A citogenética tem prestado grande subsídio, neste sentido, não só para a disciplina de genética, mas também para outras áreas. Atualmente, a genética oferece os seguintes benefícios:

a) o esclarecimento diagnóstico de quadros dismórficos observados no período neonatal, através da realização de um estudo cromossômico; b) o grande auxílio nas análises de medula óssea e sangue, especialmente nas leucemias, mas também em outros tipos de câncer;

c) a possibilidade de elucidação e confirmação diagnóstica, através da pesquisa do sítio frágil do cromossomo X e de quebras cromossômicas, de grande valia quando não se dispõe de biologia molecular para o diagnóstico de certeza;

d) o diagnóstico intra-útero das aberrações cromossômicas, por meio de exames do líquido amniótico e vilosidade corial;

e) o auxílio prestado nos distúrbios da diferenciação sexual, ditando inclusive condutas, como a extirpação de uma gônada, nas situações 
em que a presença de um cromossomo $\mathrm{Y}$ oferece riscos para $\mathrm{O}$ desenvolvimento de um gonadoblastoma;

f) a identificação da origem de cromossomos marcadores, impossíveis de serem identificados antes do advento da citogenética molecular;

g) o aconselhamento genético, que permite constatar novos modos de herança não tradicionais, como a herança mitocondrial, a dissomia uniparental ou o imprinting genômico, processos antes desconhecidos.

É possível afirmar que, após o emprego da biologia molecular, nenhuma área da biomedicina permaneceu intocada. Entretanto, o rápido desenvolvimento da biologia molecular não foi completamente absorvido pela sociedade. Assim, atualmente, há uma grande preocupação com os aspectos médico-legais e éticos decorrentes do impacto provocado pelos avanços da biologia molecular, envolvendo até mesmo as companhias de seguros (1-4).

As ações de saúde voltadas para diagnóstico precoce, o a estimativa do risco de recorrência, a identificação do grupo de risco e o aconselhamento genético (que informa e orienta o afetado e seus familiares e estabelece propostas adequadas e viáveis para cada caso) só podem beneficiar toda a coletividade e agilizar o sistema de saúde como um todo. Na medida em que estas ações abreviam o período gasto na formulação de um diagnóstico e o tempo médio de internação, antecipando um tratamento adequado e impedindo ou minimizando possíveis seqüelas, reduzem o custo hospitalar.

Na América Latina, a genética médica está atrasada em relação ao mundo industrializado. Há serviços privados disponíveis apenas para uma pequena parcela da população; a maioria não tem acesso aos serviços preventivos e assistenciais básicos de genética (5). No Estado de São Paulo, estima-se, na estrutura do serviço público, a necessidade de cerca de 20 centros especializados em genética, de complexidade terciária (1 para cada 2 milhões de habitantes), para o manejo e diagnóstico das enfermidades genéticas; além disso, estima-se que sejam necessários 400 geneticistas clínicos (1 para cada 100000 habitantes) para atendimento ambulatorial e aconselhamento genético (6). Dos 6386 hospitais do sistema público de saúde brasileiro, 149 são universitários. Entretanto, em todo o território nacional, os serviços públicos de genética médica não chegam a 40 (6).

Assim, a existência de poucos centros especializados de genética no país acarreta uma sobrecarga no serviço público e, até mesmo, no serviço privado. Este fato dificulta o encaminhamento de pacientes portadores de uma determinada anormalidade genética para atendimento especializado. A rigor, todas as doenças mencionadas aqui, e muitas outras, não citadas, deveriam passar por uma avaliação genética; só o geneticista dispõe de subsídios para esclarecer o risco para os indivíduos e para os membros de sua família, assim como para propor uma terapia antenatal, neonatal ou futura, de forma a minimizar seqüelas, propiciar melhor evolução do paciente e reduzir sobremaneira o gasto público. Neste sentido, a disponibilidade de um serviço de genética em hospitais deveria ser uma questão de interesse público.

Entre os hospitais públicos no Município de São Paulo, o Hospital Infantil Menino Jesus (HIMJ), um hospital municipal pediátrico de nível terciário, contou, até 1996, com um serviço de genética. Naquele ano, o serviço foi extinto por ocasião da implantação, pela prefeitura de São Paulo, do Plano de Atendimento à Saúde (PAS). O PAS previa a transferência do controle dos hospitais da prefeitura para cooperativas formadas por médicos e funcionários licenciados do serviço público. Esse programa acarretou profundas mudanças no sistema de saúde de toda a rede municipal e terminou por extinguir o setor de genética do HIMJ, dentre outros serviços. Entre 90 e 100 pacientes eram atendidos, por mês, neste serviço. Infelizmente, a tentativa de melhor atender às necessidades populacionais do Município através do
PAS foi infrutífera e o plano foi oficialmente extinto em agosto de 1999, comprometendo-se o governo municipal, na atualidade, a reformulá-lo.

Dessa forma, o objetivo do presente estudo foi realizar uma análise retrospectiva do trabalho do serviço de genética do HIMJ, com o intuito de ressaltar a importância de serviços desse tipo em nosso país. Além disso, o estudo pretende chamar a atenção para o fato de que atos governamentais divorciados de uma política adequada de saúde podem, a longo prazo, causar prejuízos à população.

\section{MATERIAIS E MÉTODOS}

O HIMJ é um hospital municipal pe diátrico de nível terciário. $\mathrm{O}$ setor de genética do hospital contava, entre 1992 e 1996, com duas geneticistas (uma, a partir de 1995), uma bióloga e, posteriormente, uma estagiária, no setor de citogenética.

Cada uma das duas geneticistas exercia uma atividade didático-assistencial, constituída pelo atendimento de uma média de quatro pacientes, em nível ambulatorial, por dia, três vezes por semana, sendo dois casos novos e dois retornos. Eram realizadas, também, avaliações solicitadas por outros especialistas, pela enfermaria, o berçário, isolamento, o pronto-socorro e a unidade de tratamento intensivo (UTI) (cerca de 96 casos / mês).

$\mathrm{O}$ atendimento não se limitava à esfera municipal, abrangendo, também, as solicitações de outros hospitais e postos de atendimento federais e estaduais. A média de espera para a primeira consulta era de cerca de 3 a 4 meses, gerando uma demanda reprimida. Na época, havia cerca de 12 centros de atendimento em genética na Cidade de São Paulo, para uma população de aproximadamente nove milhões (levando em conta somente a população da Cidade de São Paulo no período do estudo).

A solicitação de cariótipo só podia ser realizada por uma das geneticistas, pelo endocrinologista e pelos responsáveis pelos berçários do HIMJ e do Hospital e Maternidade Vila Nova Ca- 
choeirinha (HMVNC), também da rede municipal, em casos para os quais não houvesse possibilidade de uma avaliação em nosso ambulatório. Visávamos, com isso, observar se, de fato, havia indicação para a realização de um exame tão dispendioso e difícil como esse. Algumas vezes, outros profissionais ou serviços solicitavam o exame; porém, com raríssimas exceções, todo paciente encaminhado para avaliação ou exame passava pela triagem prévia de uma das geneticistas. As normas do setor de genética do HIMJ eram remetidas ao serviço requisitante, para que a compreensão do significado do exame de cariótipo ocasionasse melhor racionalização desse pedido. Manteve-se um intercâmbio salutar e proveitoso com outros centros de genética. Sempre que possível, a abordagem foi multidisciplinar, englobando médicos e outros profissionais da área da saúde do HIMJ.

Após o encerramento das atividades do setor, o governo municipal não permitiu a consulta aos prontuários dos pacientes do serviço; tal fato acarretou a exclusão, ou a não inclusão, de alguns pacientes, por absoluta falta de dados. Porém, a presente amostra de 571 casos que puderam ser codificados em um banco de dados (Excel 6.0) é significativa do trabalho do serviço.

Os cariótipos cujos resultados serão descritos no presente estudo foram realizados em cultura de células de sangue periférico, tanto por coloração convencional, como por diferentes técnicas de bandeamento, e em outros tecidos (fibroblastos e gônadas), quando necessário (por exemplo, em um caso de tetrassomia $12 p$ e em outro com hermafroditismo verdadeiro). $\mathrm{O}$ sítio frágil $X$ também foi pesquisado em pa cientes suspeitos de serem portadores desta síndrome.

\section{RESULTADOS}

Foram codificados, no período entre novembro de 1992 e fevereiro de 1996, 571 casos, dentre todos os avaliados no setor de genética do HIMJ, sendo solicitados 358 cariótipos. Oito não puderam ser concluídos por más condições da amostra (três casos); óbito do solicitante (dois casos); não comparecimento (um caso); e repetição da cultura por falta de crescimento (dois casos).

Cerca de $77 \%$ dos cariótipos foram solicitados por uma das geneticistas; $12 \%$ pelo endocrinologista; $6 \%$ pelo HMVNC e $4 \%$ por outros profissionais ou serviços. Ainda que não solicitado diretamente por uma das geneticistas, o caso sempre foi discutido com uma delas, a não ser em raríssimas exceções, nas quais o material a ser analisado era remetido ao serviço sem consulta prévia.

Dos 350 cariótipos realizados, 71 (20\%) mostraram alguma anormalidade cromossômica (tabela 1). Além

TABELA 1. Anormalidades cromossômicas detectadas no período de novembro de 1992 a fevereiro de 1996, Hospital Infantil Menino Jesus, São Paulo (SP), Brasil

\begin{tabular}{|c|c|c|c|}
\hline Cromossomo & Cariótipo & No. & Total \\
\hline \multirow[t]{4}{*}{21} & $46, X Y, t(21 ; 21)$ & 1 & 22 \\
\hline & $47, X Y,+21$ & 10 & \\
\hline & $47, X X,+21$ & 10 & \\
\hline & $47, X Y, \operatorname{inv}(9),+21$ & 1 & \\
\hline \multirow[t]{3}{*}{18} & $46, \mathrm{XY}, \mathrm{r}(18)$ & 1 & 7 \\
\hline & $47, X Y,+18$ & 3 & \\
\hline & $47, X X,+18$ & 3 & \\
\hline \multirow[t]{3}{*}{13} & $46, X X / 47, X X,+13$ & 1 & 3 \\
\hline & $47, X X,+13$ & 1 & \\
\hline & $47, X Y,+13$ & 1 & \\
\hline \multirow[t]{13}{*}{ Sexuais } & $45, X$ & 1 & 15 \\
\hline & $45, X, 15 p+$ & 1 & \\
\hline & $45, X / 46, X, i(X q)$ & 1 & \\
\hline & $45, X / 46, X, \operatorname{idic}(X)$ & 1 & \\
\hline & $45, \mathrm{X} / 46, \mathrm{X}, \mathrm{r}(\mathrm{X})$ & 1 & \\
\hline & $45, X / 46, X, r(X q)$ & 1 & \\
\hline & $45, \mathrm{X} / 46, \mathrm{X}, \mathrm{r}(\mathrm{Y})$ & 2 & \\
\hline & $45, X / 46, X Y$ & 1 & \\
\hline & $45, X / 46, X Y / 47, X X Y$ & 1 & \\
\hline & $45, \mathrm{X} / \mathrm{t}(4 ; 14)$ & 1 & \\
\hline & $46, X, i(X q)$ & 1 & \\
\hline & $47, X X X$ & 1 & \\
\hline & $47, X X Y$ & 2 & \\
\hline X Frágil & $46, X Y, \operatorname{fra}(X)(q 27.3)$ & 1 & 1 \\
\hline \multirow[t]{23}{*}{ Outros autossomos } & $46, X Y, \operatorname{del}(3)(q 22 q 25)$ & 1 & 23 \\
\hline & $46, X X, 4 q+$ & 1 & \\
\hline & $46, X Y, 4 q-$ & 1 & \\
\hline & $46, X X, 5 p-$ & 1 & \\
\hline & $46, X Y, \operatorname{del}(5 q)$ & 1 & \\
\hline & $46, X X, 9 q+$ & 1 & \\
\hline & $46, X Y, 9 p+$ & 1 & \\
\hline & $47, X X$, iso(9p) & 1 & \\
\hline & $46, X Y, \operatorname{inv}(11)(p 12 q 23)$ & 1 & \\
\hline & $46, X Y / 47, X Y, i(12 p)$ & 1 & \\
\hline & 47,XX,+del(15)(q2qter) & 1 & \\
\hline & $47, X X,+22 q$ & 1 & \\
\hline & $46, \mathrm{XY}, \mathrm{t}(1 ; 4)$ & 1 & \\
\hline & $45, \mathrm{XY}, \mathrm{t}(4 ; 14)$ & 1 & \\
\hline & 46,XY,der(4),t(4;10),del(10) & 1 & \\
\hline & $46, X X,-18,+t(4 p ; 18) m a t$ & 1 & \\
\hline & $46, X X, 4 p-,-8,+t(4 p ; 18)$ & 1 & \\
\hline & $46, X X,-5,-18,+\operatorname{der}(5),+\operatorname{der}(18), 5 p-, 10 q+$, mat & 1 & \\
\hline & $46, \mathrm{XX},-5,-10,+\operatorname{der}(18), \mathrm{t}(5 ; 10 ; 18)$ & 1 & \\
\hline & 46,XX,der(5),t(p15;q21),del(5)(q21-qter) & 1 & \\
\hline & $46, X X, t(11 ; 22)$ & 1 & \\
\hline & $46, X Y, \operatorname{inv}(9), t(11 ; 22)(22 q-)$ & 1 & \\
\hline & $46, X Y,-15,+t(9 p ; 15 q)$ & 1 & \\
\hline Total & & & 71 \\
\hline
\end{tabular}


TABELA 2. Freqüência de algumas enfermidades genéticas estudadas, Hospital Infantil Menino Jesus, São Paulo (SP), Brasil, 1992 a 1996

\begin{tabular}{lc}
\hline \multicolumn{1}{c}{ Categoria } & $\%(n=571)$ \\
\hline Autossômicas recessivas & 12 \\
Autossômicas dominantes & 12 \\
Ligadas ao X & 1 \\
Multifatoriais & 31 \\
Ambientais & 5 \\
Não classificadas & 23 \\
\hline
\end{tabular}

das aberrações cromossômicas, outras enfermidades genéticas foram estudadas naquele período. As freqüências de algumas delas (ressalvando-se que não conseguimos recuperar todos os dados a contento) podem ser observadas na tabela 2.

Além das lesões lábio-palatais (8\%), os defeitos de fechamento do tubo neural e uma série de outras malformações congênitas puderam ser avaliados e seguidos em nosso setor. Considerando-se os familiares até o terceiro grau, detectamos recorrência (outros membros da família afetados pelo problema) das lesões lábio-palatais em $29 \%$ dos casos. Dessa forma, foi possível informar não só aos portadores dessas lesões, como a todos os familiares envolvidos, o risco a que estavam expostos.

Os teratógenos e o uso de abortivos foram estudados de forma ativa (7-10). Em cerca de 7\% dos casos, houve referência da utilização de álcool durante a gestação, observandose os efeitos do álcool sobre o feto em $1 \%$ e a síndrome completa em $0,30 \%$. As síndromes de Noonan (1\%) e Williams (1\%) também foram detectadas, tendo sido avaliados dois irmãos, cuja mãe e avó materna também eram portadoras. Pouco mais da metade das alterações cromossômicas observadas envolveram os cromossomos 21 e sexuais. A síndrome de Down ocorreu em $4 \%$ dos casos codificados.

Para que pudéssemos completar o estudo de alguns casos, e buscando maior integração e cooperação, realizamos contatos com outros centros de genética. Como os portadores de doenças genéticas freqüentemente apresentam vários sistemas acometidos ou malformados, procuramos também informar a todas as especialidades envolvidas no caso as intercorrências atuais ou futuras que esses indivíduos poderiam vir a apresentar.

\section{DISCUSSÃO}

O artigo 196 da Constituição Federal brasileira (11) prevê que "a saúde é um direito de todos e um dever do Estado, garantido mediante políticas sociais e econômicas que visem à redução do risco de doença e de outros agravos e ao acesso universal e igualitário às ações e serviços para sua promoção, proteção e recuperação".

Entretanto, estas normas nem sempre são respeitadas, como no caso da falta de acesso da população a serviços públicos de genética médica. É indiscutível que os problemas congênitos e genéticos contribuem significativamente para a morbidade e mortalidade e, como tal, deveriam receber a devida atenção.

Nos Estados Unidos, no ano de 1980, $55 \%$ dos óbitos foram conseqüência de afeç̧ões causadas ou predispostas geneticamente, acarretando um custo de 100 bilhões de dólares (12). Ainda naquele país, a Academia Americana de Pediatria estima que entre 21 e $24 \%$ dos indivíduos abaixo de 17 anos têm uma ou mais afecções que lhes conferem alguma desvantagem. Se acrescentarmos a estes valores os $22 \%$ devidos a causas genéticas, constata-se a alta proporção de casos admitidos nos hospitais cuja causa básica é de ordem genética. Porém, a falta de pessoal treinado e de visão, por parte dos responsáveis, faz com que essas crianças e seus familiares não recebam um aconselhamento genético adequado.

A maioria dos países latino-americanos não apresenta uma política de saúde explícita e de apoio financeiro para a prevenção e tratamento de distúrbios genéticos. Falta integração entre os programas de genética e os serviços gerais de assistência primária de saúde e, principalmente, falta interação entre os médicos geneticistas e a própria comunidade médica e de saúde pública (13). Dessa forma, Penchaszadeh (13), em 1993, fez recomendações relativas ao estabelecimento de serviços de saúde genética na comunidade, contemplando a racionalização dos recursos materiais e humanos disponíveis em cada país.

Neste sentido, o serviço de genética do HIMJ, ainda que muito distante da perfeição, procurou reunir os servidores públicos municipais interessados em mais do que a simples manutenção de um emprego público. Evidentemente, não foram poucos os contratempos, mas tudo indica que o espírito construtivo de quem conviveu ao nosso lado e soube compreender as dificuldades próprias de um serviço desvinculado de uma Universidade resultou em um saldo extremamente positivo.

Assim, vários casos foram estudados no setor de genética do HIMJ (7-10). O caráter multidisciplinar de diversos grupos, por exemplo, o de intersexo, propiciou maior efetividade, eficácia e eficiência dos objetivos propostos. Em determinados distúrbios, foi possível estabelecer o prognóstico, a terapêutica e o manejo adequados antes que surgissem complicações ou lesões irreversíveis; os casos eram analisados por um grupo de profissionais especializados, entre os quais se destacava a presença do geneticista, justamente por conhecer de uma forma mais global o distúrbio genético em questão.

A presença de um serviço de genética em centros médico-hospitalares, além de minimizar as manifestações clínicas dos portadores de distúrbios genéticos, de melhorar sua qualidade de vida, bem como a de seus familiares, e de disponibilizar informações sobre o risco de recorrência, também evita que ocorram erros de encaminhamento e desperdícios. Um exemplo, ocorrido no HIMJ, foi a solicitação, por parte de outro hospital, para que realizássemos um cariótipo por suspeita de síndrome de Turner quando, na verdade, a avaliação genética revelou que o caso se tratava de torcicolo congênito. 
Nossos resultados, evidenciando $69 \%$ de outras aberrações cromossômicas que não a síndrome de Down (mais facilmente diagnosticada por um não geneticista do que os outros distúrbios genéticos), também justificam a necessidade desse tipo de especialidade em centros médico-hospitalares. Um neonatologista bem treinado talvez possa diagnosticar os três tipos de trissomias mais comumente observados e que, em nossa casuística, significaram $45 \%$ das anormalidades cromossômicas observadas; entretanto, seria isso suficiente? Será que um médico bem treinado, mas não geneticista, poderia diagnosticar e proceder a um aconselhamento genético adequado aos outros distúrbios genéticos não tão facilmente detectados no período neonatal? Como abordar os portadores da síndrome de Down?

Não se pretende, aqui, negar a importância das principais doenças que assolam o país (15), nem tampouco descaracterizar um sistema de prioridades. Pretendemos, apenas, manifestar o nosso desejo de que certas questões não andem divorciadas da política, fato já comprovado em uma experiência de 20 anos no serviço de genética da Universidade Federal da Bahia (16). Entre 1969, quando foi criado, e 1989, procurou-se integrar esse serviço de genética ao sistema de saúde pública. Entretanto, mudanças administrativas importantes reduziram-no a um pequeno consultório particular de genética - o único no Estado da Bahia. Isto releva, em última análise, a fragilidade dos sistemas de saúde pública e de educação no Brasil, à época do relato (16). Infelizmente, 10 anos depois do relato da Bahia, diante do ocorrido no HIMJ, a mesma problemática se faz sentir em nosso país, revelando a debilidade de nossas políticas de governo em relação à saúde pública e aos programas a longo prazo nessa área.
Nem sempre as políticas de saúde de um país atendem aos objetivos a que se destinam, sendo imprescindível o posicionamento dos diferentes segmentos da população diante das ações governamentais. Só dessa forma o governo poderá melhor conhecer e apreciar uma determinada situação fática, bem como seus efeitos, obtendo com isso o subsídio necessário para que suas proposições sejam mais adequadas e atendam às necessidades populacionais.

Agradecimentos. A todos os funcionários do Hospital Infantil Menino Jesus e de outras instituições, pela enorme contribuição na elevação consistente da qualidade do serviço público prestado à população carente, na época em que esse trabalho foi realizado. Agradeço ainda o incentivo da Professora Waldelice Lago Ribeiro dos Santos, fundamental para que esse estudo fosse publicado.

\section{REFERÊNCIAS}

1. Clotet J. Bioética como ética aplicada e genética. Bioética 1997;5(2):173-183.

2. Macer D. Ethics and prenatal diagnosis. Em: Milunsky A. Genetic disorders and the fetus: diagnosis, prevention and treatment. $4 \mathrm{a}$ ed. Baltimore: The Johns Hopkins University Press; 1998. p. 973-998.

3. Pelias MZ. Medicolegal aspects of prenatal diagnosis. Em: Milunsky A. Genetic disorders and the fetus: diagnosis, prevention and treatment. 4a ed. Baltimore: The Johns Hopkins University Press; 1998. p. 999-1024.

4. Wertz DC, Fletcher JC. Proposed: an international code of ethics for medical genetics. Clin Genet 1993;44:37-43.

5. Penchaszadeh VB, Beiguelman B. Serviços de genética médica na América Latina: estado atual e perspectivas - Introdução. Braz J Genet 1997;20(suppl):3 .

6. Brunoni D. O médico especialista em genética. Médicos 1999;6(janeiro/fevereiro): 49-53.

7. Albano LMJ, Luporini SM, Armando I, Mendonça ER, Correa Neto J, Ferreira AF, et al. Glicogenose tipo I assintomática e infecções de repetição. Rev Paul Ped 1993;11(4):241-245.
8. Albano LMJ, Santos JFK, Ramos PCPF, Brunoni D. Atresia de coanas: relato de um caso em que o uso do misoprostol como abortivo falhou. Rev Paul Ped 1993;11(3): 217-220.

9. Vargas FR, Brunoni D, Gonzalez CH, Kim CA, Meloni V, Conte A, et al. Investigation of the teratogenic potential of misoprostol. Teratology 1997;55:104.

10. Albano LMJ, Sato AE, Barbosa CA, Daré Jr S, Armando I. Misoprostol: relato de seis casos com malformações congênitas e um com hepatoesplenomegalia em que o uso do misoprostol falhou como abortivo. Em: Anais da V Reunião Anual da SBGC. Gramado, RS: Sociedade Brasileira de Genética Clínica; 1993. p. 19.

11. Brasil, Constituição Da República. Título VIII - da ordem social; capítulo II — da seguridade social; seção II — da saúde; artigo 196. 7a ed. São Paulo: Editora Atlas; 1988. p. 120-121.

12. Opitz JM. Prevalência de distúrbios condicionados geneticamente. Em: Opitz JM. Tópicos recentes de genética clínica. Rio de Janeiro: Sociedade Brasileira de Genética; 1984. p. 23-36.
13. Penchaszadeh VB. Establecimiento de servicios integrales de genética en los países en desarrollo: América Latina. Bol Oficina Sanit Panam 1993;115(1):39-47.

14. Brasil, Conselho Federal de Medicina. Regulamentação de planos de saúde prejudica médicos e pacientes. Jornal do Conselho Federal de Medicina 1998 junho:20-21.

15. Organización Panamericana de la Salud. Las condiciones de salud en las Américas, Brasil. Washington, DC: OPS; 1994. p. 87-100. [Publicação Científica no 549].

16. Souza MGF, Azevedo ES. Servicios de genética en las comunidades del Estado de Bahia, Brasil. Bol Oficina Sanit Panam 1993; 115(1):19-23

Manuscrito recebido em 12 de abril de 1999. Aceito em versão revisada em 13 de outubro de 1999. 
ABSTRACT The availability of genetics services in hospitals should be an issue of public concern. Having genetics services helps shorten the time spent in making diagnoses

The importance of genetics in the public health care system: Report on the closing down of a genetics division in São Paulo (SP), Brazil and reduces the average number of inpatient days; it also helps accelerate the choice of adequate treatments, prevents or minimizes sequelae, and, ultimately, reduces costs. The objective of the present study was to describe the closing down in 1996 of the genetics division at the Menino Jesus Children's Hospital, a pediatric institution located in the city of São Paulo, SP, Brazil. A retrospective analysis was carried out of the work performed by this division between 1992 and 1996, with an emphasis on the detection of chromosomal abnormalities. Of all cases assessed during the study period, 571 were entered into a database. Some kind of chromosomal abnormality was observed in $20 \%$ of the 350 karyotypes performed. The existence of genetics services in hospitals helps minimize the appearance of clinical symptoms in carriers of genetic abnormalities, improves the quality of life of these patients, and enables them to receive information regarding risk of recurrence, while preventing the waste of resources that results from tests that are costly and unnecessary. Such benefits amply justify the investment in setting up genetics services of the type described here. 